\title{
A NOTE ON PHOTOGRAPHY
}

THE PHOTOGRAPHS IN THIS BOOK are the result of two periods of collaboration with Sean Sprague, a Canadian photographer. The first series, rapidly shot over ten days in late 2010, is featured in brief photographic chapters that initiate each of the main parts of the book. The short opening chapters, acting as interludes, preview each part's main arguments and transition between worksites on the route from pigs to pork. This first series of photographs features very few human workers, effectively depicting one kind of managerial ideal of total automation without the messy contingencies of human engagement (see chapter 2). Put differently, the focus of this series on the serene environments and aesthetics of pork production should be viewed itself as a kind of capitalist dream image of the factory farm.

Selections from the second series, a set of images compiled over three months in 2013, are featured mainly at the opening of each part. Each of these images is composed from more than one thousand separate photographs, and then, through a laborious process, digitally stitched together to give the appearance of a single large-scale shot (see, for examples, figures 3.1, 5.1, 7.1, or 7.2). In a sense, the scenes in these images never happened: each one is a composite of action unfolding in place over hours. From another perspective, however, they are more realistic and detailed as a representation of these scenes of labor than one can achieve by taking a single image at a moment in time (see Butet-Roch 2015).

Although I allow these images to evoke their own impressions of modern meat making-accompanying and even standing in tension with the text- 
they should not be viewed as documentary in nature. They are conditioned by the sites that managers allowed Sprague to depict, how (or whether) those managers prepared sites prior to our arrival, the poses and tasks that some workers felt worthy of depiction over the course of hours, and the process itself generated reflections that result from self-consciously slowing down tasks. The presence of the fraught camera in these kinds of hidden spaces, where meat lobbies' efforts to combat undercover exposés have made it illegal in some states to visually depict agribusiness without an owner's permission (see Pachirat 2011), also sometimes unveiled new ways of sensing work. To this end, my brief and sporadic comments on images focus on what their execution opened up and made apparent during the photographic process.

It was what happened after these images were completed, however, that really taught me something new about how the factory farm is imagined in the public sphere. Although Sprague was solicited by junior editors to submit his images to a major photography outlet, a senior editor balked at being the first to feature them. Perhaps they just did not like the photographs. But it was their explanation that surprised me. Recounting how the news media was once engulfed in scandal after digital photographers had secretly doctored their documentary images, this editor was concerned-even if Sprague was forthright that these images are not "real" - that the subject matter was too sensitive for this kind of art. Viewers expected that a depiction of agribusiness would be purely documentary in nature. I empathize with where the editor is coming from, especially given how the camera has been pivotal to activist projects seeking to unveil the truth of factory farms. But I also take this as a minor reflection of the broader kinds of cultural work people put in to insist that things like modern slaughterhouses (and the people who occupy them) are distinctive sites that come with special rules of representation. Animal agribusiness is deemed scandalous in popular culture, a special domain, an exceptional deviation from some people's imagined norms of American capitalism and society. Rightly or wrongly, I hear in these concerns a sense that this site is unique, and all of these unspoken rules of depiction seem to insist that this is not us. What if, instead, we took these operations as not exceptional at all-as normal, and even prescient, reflections of American industrial worlds to come in the near future? 